\title{
Avoiding overdiagnosis in lung cancer screening: the volume doubling time strategy
}

\author{
Marie-Pierre Revel ${ }^{1,2}$
}

Affiliations: ${ }^{1}$ Université Paris Descartes, Sorbonne Paris Cité, Paris, ${ }^{2}$ Assistance Publique Hôpitaux de Paris, Paris, France.

Correspondence: M-P. Revel, APHP, Hotel Dieu, 1 Place du Parvis de Notre Dame, 75004 Paris, France. E-mail: marie-pierre.revelahtd.aphp.fr

0 @ERSpublications

Volumetry-based management of nodules observed with screening succeeds in omitting indolent, slow-growing cancers http://ow.ly/pkHY7

It has been 2 years since the breakthrough publication of the National Lung Cancer Screening Trial (NLST) results, yet there are still unresolved questions about cancer screening. Despite the possibility of reducing lung cancer-related mortality, the risk of detecting indolent lesions that are not life threatening, especially in advanced-age patients, still exists. Another problem is the number of false-positive findings leading to unnecessary diagnostic work-ups. In the NSLT study, for example, $27.2 \%$ of computed tomography participants were false positives, leading to follow-up diagnostic procedures in $90 \%$ of cases and to surgery in $4.2 \%$ [1].

Of course, computed tomography protocols and criteria for screening positivity should ensure accurate detection of all potentially malignant lesions, but this goal alone is not sufficient. Ideally, screening protocols should allow us to detect only true lung cancers, leaving out benign lesions, and among the malignant lesions detected, we should only be identifying those that are sufficiently aggressive to be potentially life threatening.

This issue of the European Respiratory Journal includes two articles addressing precisely these problems. The paper by INFANTE et al. [2] is a review article dedicated to the slow-growing lung cancer entity, whereas the paper from HOREWEG et al. [3] gives an overview of the NELSON strategy's performance, especially its rate of false positives.

\section{Do slow-growing lung cancers exist?}

INFANTE et al. [2] provide evidence of slow-growing lung cancers by analysing tumour volume doubling times (VDTs) in different lung cancer series. Considering a VDT $>400$ days to define slow-growing lung cancers, they report a highly variable proportion, ranging from 3\% in the International Early Lung Cancer Action Program (I-ELCAP) [4] to 45\% in some Japanese series [5]. The authors emphasise that only a minority of baseline VDTs were assessed in I-ELCAP, but there are other explanations as well. Differences in the screening method (chest radiography or computed tomography) must be taken into account, along with differences regarding the sex and ethnicity of the screened population. Series with a high proportion of slow-growing cancers include not only males but also females or, potentially, more patients with subsolid lesions [6]. The term subsolid refers to: 1) pure ground-glass (e.g. nonsolid) nodules (pGGNs), through which normal parenchymal structures can be visualised; and 2) part-solid nodules (or mixed ground-glass opacities (GGO)), combining ground glass and solid components.

Received: Sept 082013 | Accepted after revision: Sept 252013

Conflict of interest: None declared.

Copyright (C)ES 2013 
It is well known that the VDT of malignant lung nodules is highly dependent on their nonsolid component proportion, with the highest values hovering around 800 days for pGGNs [7, 8].

The Japanese series provide greater understanding of the correlations between subsolid lesions and pathological features in lung adenocarcinoma [9]. The nonsolid component usually reflects in situ (formally bronchioalveolar carcinoma) or minimally invasive adenocarcinoma, according to the revised classification of adenocarcinomas, whereas the solid part usually reflects an invasive component [10-13]. It is even possible to detect precancerous lung adenocarcinoma lesions, such as atypical adenomatous hyperplasia, while at the same time increasing the likelihood of indolent lesion overdiagnosis.

According to INFANTE et al. [2], it is difficult to accurately appraise the magnitude of slow-growing lung cancer phenomena, as VDTs are infrequently reported for baseline tumours. An alternative method could be to simply analyse the proportion of subsolid lesions, especially pGGNs, among the screen detected nodules.

This information, which is not always available, is provided by HOREwEG et al. [3] for the NELSON trial. In three screening rounds, the scans detected a total of 31683 nodules; $266(0.8 \%)$ were part-solid and 298 $(0.9 \%)$ nonsolid. The proportion of subsolid nodules was $1.8 \%$ on a per-nodules basis and $2.3 \%$ on a perscan basis, which is consistent with another recent series [13].

The risk of computed tomography screening selecting an excess of slow-growing cancers cannot be ignored and the NLST results bear this out. More bronchoalveolar (in situ) carcinomas and adenocarcinomas were found in the low-dose computed tomography group than in the radiography group, whereas the frequencies of other histological features were similar [1].

How to reduce false-positive computed tomography results

This is a major concern in lung cancer screening studies, negatively impacting, at least temporarily, the quality of life of screened patients [14].

Advances in computed tomography technology now allow us to obtain high-resolution images of a submillimetre thickness, which increases small nodule detection. When using $10-\mathrm{mm}$ thick images, such as in the first ELCAP study, the proportion of patients with at least one noncalcified nodule was $23 \%$ and reached $45 \%$ in the French study DepiSCAN, using 1-mm thick images [15]. Since every noncalcified nodule was considered a positive screen result in the DepiSCAN study, only $5 \%$ of the positive screens were true positive.

Indeed, the rate of false positives strongly depends on the criteria defining a positive screen. In 2005, the Fleischner Society published guidelines in order to reduce the number of follow-up computed tomographies performed for indeterminate solid nodules [16]. However, radiologists' adherence to these guidelines is unsatisfactory at best, with a tendency towards over-management [17-19]. Some new morphological criteria could help us to reduce unwarranted follow-ups.

Nodules classified as perifissural can be considered benign, as proven by long term follow-up, even in the case of rapid growth in high-risk patients $[20,21]$. These nodules probably correspond to perifissural lymph nodes and represented $20 \%$ of the nodules found at baseline screening in the NELSON study, a result that is far from insignificant [21].

Conversely, we know now that the 2-year stability concept is not valid for excluding malignancy in subsolid lesions. Patient follow-up must be extended to $\geqslant 3$ years and perhaps never be interrupted [22-24].

Along with these advances in knowledge, new tools to improve detection and characterisation of nodules have been developed. Developing computer-aided detection (CAD) tools in order to enhance small lesion detection might seem paradoxical, as computed tomography already detects too many nodules. Nonetheless, human error does occur, which is why most screening studies require double readings, except for the NLST. Radiologist performance can be significantly improved by CAD, as the NELSON group reported $78 \%$ sensitivity for nodule detection with double reading and $96.7 \%$ with CAD [25]. Excluding nodules $<5 \mathrm{~mm}$ reduces false-positive detections to an acceptable mean number of 1.9 per examination. Still, CAD systems are not sensitive enough to detect subsolid nodules and large spiculated masses [26]. The time for software to replace radiologists has not yet come.

Software will also automatically calculate VDT, which, most notably, helps indeterminate nodule management $[27,28]$. This strategy, chosen by the NELSON group, reduces the proportion of positive screens while increasing the proportion of true positives. HOREwEG et al. [3] provide both the lung cancer detection rate and the false-positive rate of the NELSON trial. 5.5-year lung cancer risk calculations are also provided and markedly vary according to baseline computed tomography results, from $1 \%$ after a negative baseline scan to $48.3 \%$ after a positive baseline scan. These data should help to counsel patients eligible for screening. 
The cumulative lung cancer detection rate is comparable to that of the NLST $(2.6 \%$ and $2.4 \%$ in NELSON and NLST trials, respectively) but there is a major difference in the rate of false positives $(3.6 \%$ versus $27.2 \%)$ and of unnecessary surgeries ( $0.8 \%$ versus $4.2 \%)$, on a per-participant basis. HoreweG et al. [3] do not comment on another potential harm from screening, which is over-diagnosis. Because the NELSON strategy is mainly based on volumetric growth assessment, indolent cancers exhibiting no growth or very long doubling times are not considered as positive screens and are not referred to pulmonologists for a diagnostic work-up, which largely reduces the risk of overdiagnosis.

Does positron emission tomography help when dealing with positive screen results? We can take issue with INFANTE et al. [2] on one point: the idea that fluorodeoxyglucose positron emission tomography (PET) is a key technique for differentiating benign and malignant nodules, in a screening context. For instance, two meta-analyses in a nonscreening context arrived at very different conclusions concerning the superiority of PET over computed tomography [29, 30]. INFANTE et al. [2] do mention the prospective study by FLETCHER et al. [31] reporting a higher specificity of PET for diagnosing malignancy. Nevertheless, the study was based on nodules found on chest radiography, which selects larger nodules than those detected on computed tomography accounting for the very high proportion of malignancy in this series.

In the third edition of its guidelines, the American College of Chest Physicians (ACCP) recommends performing PET for solid nodules $>8 \mathrm{~mm}$ in diameter with a low-to-moderate pretest probability of malignancy [32]. However, such nodules represent only a small minority of the nodules found on screening; $41-57 \%$ of the nodules found at baseline computed tomography screenings measured $<5 \mathrm{~mm}$ in the ELCAP cohorts and $81-88 \%$ measured $<10 \mathrm{~mm}$. The proportion $<8 \mathrm{~mm}$ is not mentioned [33].

PET is usually negative in malignant subsolid nodules and may be also falsely negative in small solid lung tumours, two reasons explaining the $32 \%$ false negativity in a screening context. Notably, PET was not routinely included in the work-up of positive screens in NELSON trial in which the presence or absence of growth of nodule was leading and not the outcome of the PET scan [34].

\section{How should we handle subsolid nodules?}

A proportion of these nodules are no more than transient inflammatory lesions that will spontaneously resolve, especially if they show a large solid portion and ill-defined borders, but since there are no reliable distinctive patterns between resolving and persisting ones, all must undergo a follow-up computed tomography $[35,36]$. Early follow-up at 3 months is recommended by the Fleischner Society and ACCP 2013 guidelines, "except for individuals with life-limiting comorbidities, or by others who place a high value on avoiding treatment of possibly indolent lung cancer" [23, 32].

In the NELSON trial nodule management protocol, pGGNs are not considered actionable lesions until a solid component appears or until their VDT shortens to $<400$ days [34]. The MILD (Multicentric Italian Lung Detection) trial similarly adopted a nonsurgical approach and a surveillance attitude [37]. Whereas the need to resect pGGNs and part-solid nodules, when stable, is questionable, growing subsolid lesions are more consensually referred for surgery, unless there are major comorbidities.

Detection of growth is more challenging for subsolid nodules than for solid ones. Indeed, most algorithms combine shape analysis with a first-step density-based segmentation. The process may fail to discriminate subsolid nodules from the surrounding normal lung parenchyma, due to their close density. This is why HOREWEg et al. [3] indicate the method for calculating VDTs in cases of software failure (very useful when software is not available). The formula, found on dedicated chest medicine websites, is based on the evolution of nodule diameters manually measured by the radiologist. Measurement error has been evaluated not to exceed $1.7 \mathrm{~mm}$, thus any change in diameter $\geqslant 2 \mathrm{~mm}$ is significant and was chosen to define growth in the largest recent series on growing GGO $[8,38]$.

\section{What are the risks in leaving slow-growing lung cancer untreated?}

This is what INFANTE et al. [2] discuss in the "areas of uncertainty" section of their review. The evidence given for some slow-growing lung cancers that suddenly exhibit accelerated growth is scant: INFANTE et al. [2] only mention that such phenomena have been occasionally observed in the DANTE and NELSON trials.

In large series, CHANG et al. [8] reported that $90 \%$ of pGGNs did not grow, with a median follow-up of 5 years [8]. Only one nodule progressed beyond stage I, but from an initial 5-mm pGGN, it had transformed into a 14-mm part-solid nodule by 68 -month follow-up, and was pathologically staged as N2 because of micronodal invasion. The size of the solid portion, whether the patient had annual computed tomography, and the delay between the onset on the solid component and surgery, are not mentioned. The risk of nodal spread is generally considered to be very limited with a solid proportion $\leqslant 50 \%$ [39]. 
It is thus reasonably safe to consider leaving patients with pGGN untreated on the condition they undergo an annual computed tomography to ensure no solid component appears. Although $39 \%$ of such nodules show minimal invasive pathological features, they remain a stage I disease. A cut-off value of $16.4 \mathrm{~mm}$ has been suggested to suspect invasiveness [40].

\section{What are the best therapeutic options for slow-growing lung cancers?}

INFANTE et al. [2] end their review by discussing specially tailored therapeutic options, which include surgical and nonsurgical possibilities. It is still not certain that the ongoing randomised trial comparing lobectomy with limited resection for stage I peripheral nonsmall cell lung cancer will give us all the answers we need regarding subsolid lesion management, as it excludes "radiologically determined noninvasive cancer" [41]. Stereotactic radiotherapy and radiofrequency ablation are other options, but no prospective studies compared their respective performances.

What options are there and for which patients? Management of slow-growing lung cancer cannot be standardised and all options must be discussed on a case-by-case basis. As INFANTE et al. [2] write, "decision-making rests on a careful balance of competitive risks related to the natural course of the disease, life expectancy and impact of treatment".

In conclusion, if the NELSON trial, like other European screening trials, is not statistically powerful enough to call into question the NLST results, it demonstrates that volumetry-based management of nodules observed with screening not only reduces computed tomography false positivity to a very acceptable level but also succeeds in omitting indolent, slow-growing cancers, while minimising the risk of overdiagnosis.

\section{References}

1 Church TR, Black WC, Aberle DR, et al. Results of initial low-dose computed tomographic screening for lung cancer. N Engl J Med 2013; 36: 1980-1991.

2 Infante M, Berghmans T, Heuvelmans MA, et al. Slow-growing lung cancer as an emerging entity: from screening to clinical management. Eur Respir J 2013; 42: 1706-1722.

3 Horeweg N, van der Aalst CM, Vliegenthart R, et al. Volumetric computer tomography screening for lung cancer: three rounds of the NELSON trial. Eur Respir J 2013; 42: 1659-1667.

4 Henschke CI, Yankelevitz DF, Yip R, et al. Lung cancers diagnosed at annual CT screening: volume doubling times. Radiology 2012; 263: 578-583.

5 Sone S, Hanaoka T, Ogata $\mathrm{H}$, et al. Small peripheral lung carcinomas with five-year post-surgical follow-up: assessment by semi-automated volumetric measurement of tumour size, CT value and growth rate on TSCT. Eur Radiol 2012; 22: 104-119.

6 Lindell RM, Hartman TE, Swensen SJ, et al. Five-year lung cancer screening experience: CT appearance, growth rate, location, and histologic features of 61 lung cancers. Radiology 2007; 242: 555-562.

7 Hasegawa M, Sone S, Takashima S, et al. Growth rate of small lung cancers detected on mass CT screening. $\mathrm{Br} \mathrm{J}$ Radiol 2000; 73: 1252-1259.

8 Chang B, Hwang JH, Choi $\mathrm{YH}$, et al. Natural history of pure ground-glass opacity lung nodules detected by lowdose CT scan. Chest 2013; 143: 172-178.

9 Nakata M, Saeki H, Takata I, et al. Focal ground-glass opacity detected by low-dose helical CT. Chest 2002; 121 : 1464-1467.

10 Travis WD, Brambilla E, Noguchi M, et al. International Association for the Study of Lung Cancer/American Thoracic Society/European Respiratory Society international multidisciplinary classification of lung adenocarcinoma. J Thorac Oncol 2011; 6: 244-285.

11 Austin JH, Garg K, Aberle D, et al. Radiologic implications of the 2011 classification of adenocarcinoma of the lung. Radiology 2013; 266: 62-71.

12 Lederlin M, Puderbach M, Muley T, et al. Correlation of radio- and histomorphological pattern of pulmonary adenocarcinoma. Eur Respir J 2013; 41: 943-951.

13 Henschke CI, Yankelevitz DF, Mirtcheva R, et al. CT screening for lung cancer: frequency and significance of partsolid and nonsolid nodules. AJR Am J Roentgenol 2002; 178: 1053-1057.

14 van den Bergh KA, Essink-Bot ML, Borsboom GJ, et al. Long-term effects of lung cancer computed tomography screening on health-related quality of life: the NELSON trial. Eur Respir J 2011; 38: 154-161.

15 Blanchon T, Brechot JM, Grenier PA, et al. Baseline results of the Depiscan study: a French randomized pilot trial of lung cancer screening comparing low dose CT scan (LDCT) and chest X-ray (CXR). Lung Cancer 2007; 58: 50-58.

16 MacMahon H, Austin JH, Gamsu G, et al. Guidelines for management of small pulmonary nodules detected on CT scans: a statement from the Fleischner Society. Radiology 2005; 237: 395-400.

17 Eisenberg RL, Bankier AA, Boiselle PM. Compliance with Fleischner Society guidelines for management of small lung nodules: a survey of 834 radiologists. Radiology 2010; 255: 218-224.

18 Lacson R, Prevedello LM, Andriole KP, et al. Factors associated with radiologists' adherence to Fleischner Society guidelines for management of pulmonary nodules. J Am Coll Radiol 2012; 9: 468-473.

19 Masciocchi M, Wagner B, Lloyd B. Quality review: Fleischner criteria adherence by radiologists in a large community hospital. J Am Coll Radiol 2012; 9: 336-339.

20 Ahn MI, Gleeson TG, Chan IH, et al. Perifissural nodules seen at CT screening for lung cancer. Radiology 2010; 254 : 949-956.

21 de Hoop B, van Ginneken B, Gietema H, et al. Pulmonary perifissural nodules on CT scans: rapid growth is not a predictor of malignancy. Radiology 2012; 265: 611-616. 
lung: recommended interim guidelines for assessment and management. Radiology 2009; 253: 606-622.

23 Naidich DP, Bankier AA, MacMahon H, et al. Recommendations for the management of subsolid pulmonary nodules detected at CT: a statement from the Fleischner Society. Radiology 2013; 266: 304-317.

24 Min JH, Lee HY, Lee KS, et al. Stepwise evolution from a focal pure pulmonary ground-glass opacity nodule into an invasive lung adenocarcinoma: an observation for more than 10 years. Lung Cancer 2010; 69: $123-126$.

25 Zhao Y, de Bock GH, Vliegenthart R, et al. Performance of computer-aided detection of pulmonary nodules in lowdose CT: comparison with double reading by nodule volume. Eur Radiol 2012; 22: 2076-2084.

26 Xie X, Zhao Y, Snijder RA, et al. Sensitivity and accuracy of volumetry of pulmonary nodules on low-dose 16- and 64-row multi-detector CT: an anthropomorphic phantom study. Eur Radiol 2013; 23: 139-147.

27 Revel MP, Lefort C, Bissery A, et al. Pulmonary nodules: preliminary experience with three-dimensional evaluation. Radiology 2004; 231: 459-466.

28 Revel MP, Merlin A, Peyrard S, et al. Software volumetric evaluation of doubling times for differentiating benign versus malignant pulmonary nodules. AJR Am J Roentgenol 2006; 187: 135-142.

29 Gould MK, Maclean CC, Kuschner WG, et al. Accuracy of positron emission tomography for diagnosis of pulmonary nodules and mass lesions: a meta-analysis. JAMA 2001; 285: 914-924.

30 Cronin P, Dwamena BA, Kelly AM, et al. Solitary pulmonary nodules: meta-analytic comparison of cross-sectional imaging modalities for diagnosis of malignancy. Radiology 2008; 246: 772-782.

31 Fletcher JW, Kymes SM, Gould M, et al. A comparison of the diagnostic accuracy of 18F-FDG PET and CT in the characterization of solitary pulmonary nodules. J Nucl Med 2008; 49: 179-185.

32 Gould MK, Donington J, Lynch WR, et al. Evaluation of individuals with pulmonary nodules: when is it lung cancer? Diagnosis and management of lung cancer, 3rd ed: American College of Chest Physicians evidence-based clinical practice guidelines. Chest 2013; 143: e93S-120S.

33 Henschke CI, Yankelevitz DF, Naidich DP, et al. CT screening for lung cancer: suspiciousness of nodules according to size on baseline scans. Radiology 2004; 231: 164-168.

$34 \mathrm{Xu} \mathrm{DM}$, Gietema H, de Koning H, et al. Nodule management protocol of the NELSON randomised lung cancer screening trial. Lung Cancer 2006; 54: 177-184.

35 Khokhar S, Mironov S, Seshan VE, et al. Antibiotic use in the management of pulmonary nodules. Chest 2010; 137: 369-375.

36 Lee SM, Park CM, Goo JM, et al. Transient part-solid nodules detected at screening thin-section CT for lung cancer: comparison with persistent part-solid nodules. Radiology 2010; 255: 242-251.

37 Silva M, Sverzellati N, Manna C, et al. Long-term surveillance of ground-glass nodules: evidence from the MILD trial. J Thorac Oncol 2012; 7: 1541-1546.

38 Revel MP, Bissery A, Bienvenu M, et al. Are two-dimensional CT measurements of small noncalcified pulmonary nodules reliable? Radiology 2004; 231: 453-458.

39 Lee SM, Park CM, Paeng JC, et al. Accuracy and predictive features of FDG-PET/CT and CT for diagnosis of lymph node metastasis of T1 non-small-cell lung cancer manifesting as a subsolid nodule. Eur Radiol 2012; 22: $1556-1563$.

40 Lim HJ, Ahn S, Lee KS, et al. Persistent Pure Ground-Glass Opacity Lung Nodules $>/=10$ mm in Diameter at CT: Histopathologic Comparisons and Prognostic Implications. Chest 2013 [In press DOI: 10.1378/chest.12-2987].

41 Nakamura K, Saji H, Nakajima R, et al. A phase III randomized trial of lobectomy versus limited resection for smallsized peripheral non-small cell lung cancer (JCOG0802/WJOG4607L). Jpn J Clin Oncol 2010; 40: 271-274. 